\title{
Identifying weak joints in jointed concrete pavements from TSD measurements by basis pursuit
}

\author{
M. Scavone, S.W. Katicha \& G.W. Flintsch \\ Center for Sustainable and Resilient Transportation Infrastructure, Virginia Tech Transportation Institute. \\ Blacksburg, USA
}

\begin{abstract}
Weak joints are the source of most performance problems in jointed concrete pavements (JPCP) and composite pavements created by overlaying a JPCP. Until recently, it has been prohibitively time-consuming to evaluate the joints in a road network, and the evaluation of joint load transfer efficiency (LTE) has been restricted to specific project-level applications. This is changing with the advent of the Traffic Speed Deflectometer (TSD), a device that can collect structural information data at a $1 \mathrm{~m}$ resolution while moving at traffic speed. The $1 \mathrm{~m}$ resolution is adequate to capture the response of the joints to the applied load, but it results in a higher noise level compared to data collected at lower resolutions (e.g. at the typical $10 \mathrm{~m}$ ). In this paper, we propose the use of Basis Pursuit (BP) to extract meaningful information about the joints' condition from the (noisy) TSD measurements. Weak joints are modeled as spikes (Dirac basis) in the measurements while the remaining features in the measurements are modeled by a wavelet basis. Combining the two bases (Dirac basis and wavelet basis) results in multiple possible representations of the collected measurements: we essentially have twice the number of unknowns than the number of equations to determine these unknowns. BP seeks a representation with a small number of elements from the two bases. Because weak joints are best represented by spikes, BP denoising results in mostly selecting the spikes at the weak joints location. These identified spikes provide a list of weak joints that should be further investigated (e.g. by performing LTE testing) or should receive priority in repair. We present examples of BP using simulated data and actual TSD collected data.
\end{abstract}

Keywords: TSD, concrete, joints, denoising, BP

\section{INTRODUCTION}

A major source of distress in jointed pavement structures, like jointed Portland concrete pavements (JPCP) and overlays of these, are deteriorated load-carrying joints. Deteriorated (or "weak") joints are those that lost their capacity to transfer the traffic loads from one slab to the adjacent ones - their Load Transfer Efficiency (LTE) is low (Alavi et al., 2008; Pierce et al., 2003). A jointed pavement may develop surface pathologies following the loss of LTE at its joints: the excessive movement of the slabs contributes to the pumping of the fine materials of the foundation layer, which in turn leads to joint faulting and slab cracking out of the loss of support (Huang, 2004; Delatte, 2014). Besides, if the concrete pavement is overlaid with asphalt, reflection cracking will occur on the surface layer at the location of the weak joints (Huang, 2004).

Thus, knowledge of the LTE of the joints of an in-service pavement is crucial both at the project level (input to the design of overlays or reinforcements (AASHTO, 2015)) and at the

DOI: $10.1201 / 9781003222880-39$ 
network-wide management level, where structural performance data is most advisable for proper resource allocation (Haas et al., 2015).

At the project level, the Falling Weight Deflectometer (FWD) has been the device of choice to perform LTE testing (Alavi et al., 2008; Pierce et al., 2003; AASHTO 2015; Haas et al., 2015). However, network-wide LTE testing with FWD devices is unfeasible because of the device's stop-and-go nature, which makes it unsuitable for large-scale surveys - Flintsch et al (2013) point out that an FWD can cover no more than 180 tests/workday, which translates to 18 lane-miles at a 0.1 -mile resolution. if translated to LTE testing of a jointed pavement where the joints are spaced 15 feet, and two tests are required at each location, the production rate of a single FWD drops to roughly $400 \mathrm{~m}$ ( 0.25 miles) per day. Thus, a timely periodic networkwide survey would require a large fleet of FWDs operating simultaneously on in-service roads, which is impracticable both in terms of operational costs and from a safety standpoint (Flintsch et al., 2013; Katicha et al., 2013; Haas et al., 2015; Rada et al., 2016).

Traffic-speed deflection devices (TSDDs) overcome these limitations (European Commission, 1997; Arora et al, 2006; Flintsch et al., 2013; Rada et al.; 2016) by surveying the deflection of the pavement surface (or the velocity at which the pavement deflects) non-stop and in response to the device's own weight as it travels along the test pavement. Several prototypes were built over the years (Arora et al., 2006; Flintsch et al., 2013; Rada et al., 2016; Andersen et al., 2017), but only two types of devices are currently operational: the Rapid Pavement Tester (RAPTOR) and the Traffic Speed Deflectometer (TSD) - the widely-researched Rolling Weight Deflectometer (RWD) (Steele et al., 2020) has been decommissioned as of early 2020. Plenty of published literature exists on the use of these devices for network-wide assessments, some key examples are Flintsch et al. (2013); Rada et al. (2016). In general, comparisons of these devices against traditional deflection devices like the FWD and deflectographs returned good agreement between traffic speed devices and their static counterparts, provided that the contrasts are done in terms of comparable physical magnitudes (Katicha et al., 2014; Flintsch et al., 2013). However, little published material exists on using these devices to survey networks of jointed pavements (plain concrete pavements and overlays of these). A possible reason for that is that so far they are deemed not suitable for rigid pavements for the little deflection produced under its load - in fact, the Australian Standard on the TSD for deflection surveying (Austroads, 2016) specifically advises against using this device on rigid pavements. Yet, in one of the few published case studies of rigid pavement surveying with a TSD (Flintsch et al., 2013), it was found that the TSD would be suitable as a screening tool, that is, a device to perform a first 'scan' of the entire network to detect locations with structural deficiencies worth of further investigation and/or immediate repair. Katicha et al. (2013; 2014; 2016) provide a framework to denoise the dense TSD data [1-meter resolution] and extract meaningful features for further analysis.

\section{OBJECTIVES}

In this paper, we present the use of Basis Pursuit [BP] (Chen et al., 2001) on dense TSD deflection slope measurements to remove unwanted noise and extract the pulse responses (peaks in deflection) produced by weak locations, such as weak joints in jointed pavement networks. We aim at decomposing the TSD measurements into a combination of continuous components plus pulse responses due to the weak spots. BP is an objective, reproducible, and computationally fast methodology to achieve this task, even with large input datasets. Thus, it is suitable as a framework to easily detect weak spots within a pavement segment that was surveyed at a high resolution.

The remainder of this paper is divided into three sections. Firstly, we briefly present BP as a signal processing technique, relate it to the Least Absolute Shrinkage and Selection Operator [LASSO] (Tibshirani, 1996; Chen et al.,2001; Hastie et al., 2009), and discuss practical issues related to the selection of its hyper-parameters. Following, we present a case of signal denoising with BP using a simulated dataset. Finally, we present the results of BP denoising of actual TSD signals from different backgrounds: an experimental test track and an actual 
survey of overlaid jointed pavements. This paper contains an abridged version of a recently published article (Scavone et al., 2021), yet the intention of this paper is not to dive too much into the mathematical and computational detail behind BP, but actually to focus on the applicability as a denoising technique for TSD measurements, providing an example of an actual TSD survey on composite jointed pavements that we denoised with BP.

\section{A BRIEF OVERVIEW OF BASIS PURSUIT}

Basis Pursuit [BP] (Chen et al., 2001) is a convex optimization procedure that seeks a representation of a given signal $y$ with $n$ values using the fewest components from a given signal dictionary (a collection of elementary signals forming a basis or set of bases), that is, a sparse representation of $y$. The size of the dictionary would not affect the decomposition process per se, BP can be applied to decompose $y$ over an over-complete dictionary (a dictionary with more than $n$ elementary signals), a problem with many possible solutions, and BP would return a decomposition based on few non-zero components (a sparse decomposition).

In practical terms, such over-complete dictionaries may be warranted, as a sparse representation of the signal $y$ may be achieved using sub-sets of components from multiple bases. For instance, the deflection measurements from a TSD assumed as a composition of a continuous component plus pulse responses could be described sparsely as a combination of Dirac signals (pulses) plus a combination of elementary continuous signals. Meanwhile, restricting to singlebase decomposition (either only continuous signal base or Dirac base only) is likely to lead to non-sparse representations - a remarkable example of this is the Gibbs Phenomenon.

For this application, we are modeling the pulse responses as Dirac signals and the continuous component as a combination of wavelets because most measured signals admit a sparse representation in the wavelet domain, which in turn enhances the removal of noise, and because computations involving wavelets are very fast. Each basis has $n$ components, thus our over-complete dictionary has $2 n$ components. Given a TSD signal as input, BP would return a sparse decomposition of it over the two bases: the continuous component would represent any smooth variation in deflection over the surveyed pavement while the Dirac component would contain the pulse responses produced by the weak spots, the feature we attempt to extract in order to locate them.

The BP optimization problem for a generic signal $y$ can be mathematically formulated as:

$$
\min \|\alpha\|_{1} \text { subject to } y=\phi \alpha
$$

In the equation above, $\Phi$ is an $n$-by- $2 n$ matrix containing the signal dictionary, and $\alpha$ is the vector of coefficients to reconstruct $y$ as a combination of the columns of $\Phi$. Alternatively, when the ultimate goal is to both remove the noise present in the signal $y$ and simultaneously sparsely decompose $y$, BP can be formulated as a particular LASSO regression [or $\mathrm{L}_{1}$ regularization] problem (Tibshirani, 1996; Chen et al.,2001; Hastie et al., 2009):

$$
\min _{\alpha}=\frac{1}{2}\|y-\phi \alpha\|_{2}^{2}+\lambda\|\alpha\|_{1} \text { where }: y=z+\sigma \in \text { and } \in \sim N(0,1)
$$

In the particular case of TSD signals, $z$ is the unknown true pavement deflection response, and $y$ is the noisy measurements collected with the TSD, $\sigma$ is the standard deviation of the noise in the TSD signal.

LASSO regression problems have a known solution (Tibshirani, 1996; Chen et al., 2001; Friedman et al., 2010), which obviously depends on the hyper-parameter $\lambda$ - for onedimension variables, it is the soft-thresholding function with parameter $\lambda$. Friedman et al.'s scheme to solve the LASSO problem is numeric and consists of applying the soft-thresholding function coordinate-wise at each descent step - at each iteration, the coordinates of $\alpha$ are updated one at a time. Furthermore, the fact that the matrix $\Phi$ can be thought of as a stack of 
orthonormal columns (as is the case with BP) is advantageous: the cyclic coordinate descent can be done in batch-mode, instead of updating the coordinates of $\alpha$ one by one, all the coordinates that relate to a given stack of orthonormal columns can be updated simultaneously. In the BP denoising case, where $\Phi=\left[\Phi_{1}, \Phi_{2}\right]$ and $\alpha=\left[\alpha_{1}, \alpha_{2}\right]$, where $\Phi_{1}$ is the Dirac base (and $\alpha_{1}$ is its parameters' vector) and $\Phi_{2}$ is the wavelet base (related to the vector $\alpha_{2}$ ), a single iteration of the batch coordinate descent first updates $\alpha_{1}$ while leaving $\alpha_{2}$ constant, and secondly proceeds with $\alpha_{2}$ without altering $\alpha_{1}$ until the next iteration step. Refer to Scavone et al. (in press) for more details on the procedure.

Two major trends exist on the choice of the hyper-parameter $\lambda$, these are grounded on a bias-variance trade-off decision. One possible choice for $\lambda$ is the so-called Universal Threshold (Donoho and Johnstone, 1994). The Universal Threshold is a value of $\lambda$ high enough that retains only those components of the noisy input signal that have a high probability of being in the true signal. However, using a high threshold may both ignore real signal features whose amplitude is smaller than $\lambda$ while at the same time will dampen the recovered signal excessively towards zero. Alternatively, $\lambda$ could be chosen such that the BP output signal resembles the unknown signal $z$ the most - it minimizes the error between $z$ and $\Phi \alpha$. Computationally, however, the procedure may require time, as the BP decomposition must be applied using all candidate values of $\lambda$ - yet anyway $\lambda$ is bounded by zero and the Universal Threshold, which narrows the search effort. Since $z$ is unknown (and so the fit error cannot be directly calculated), Stein's SURE estimate (Stein, 1981; Tibshirani, 1996) must be used. The formula for SURE that corresponds to optimization problems like BP is given by Tibshirani and Taylor (2012) and reproduced in Scavone et al. (2021).

\section{BASIS PURSUIT APPLIED TO SIMULATED DATA}

In this section, we provide a demonstration of the capabilities of BP as a denoising and feature extraction tool by reconstructing a simulated signal. The signal (a size-2048 vector) is a composition of a sinusoidal component with amplitude 2 , centered around zero, and wavelength of 400 plus a discontinuous signal composed of peaks of amplitude 7 every 50 units (Figure 1). We added random noise to the simulated signal (normally distributed, with mean zero and variance 1), and attempted to reconstruct the true signal as a composition of pulses and wavelets. In this demonstration, we reconstructed the noisy signal by both $\mathrm{BP}\left(\mathrm{L}_{1}\right.$ regularization) and Ridge regression ( $\mathrm{L}_{2}$ regularization). Figure 1 portrays the true and noisy signals, and Figure 2 presents the reconstruction results for both regularization techniques for the value of $\lambda$ that minimizes the reconstruction error. Moreover, Figure 3 shows a plot of the coefficients that correspond to both bases according to both signal recovery methods.

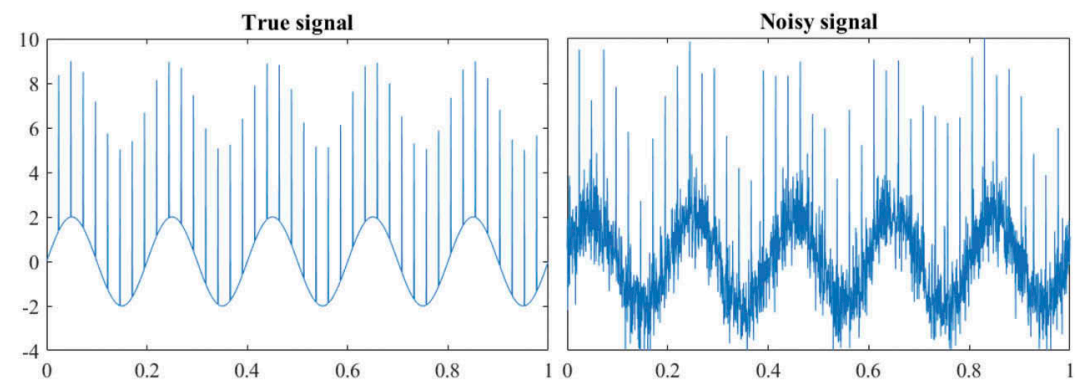

Figure 1. Simulated signal crafted for the BP application example. Left: generated signal, right: signal with added random noise. 

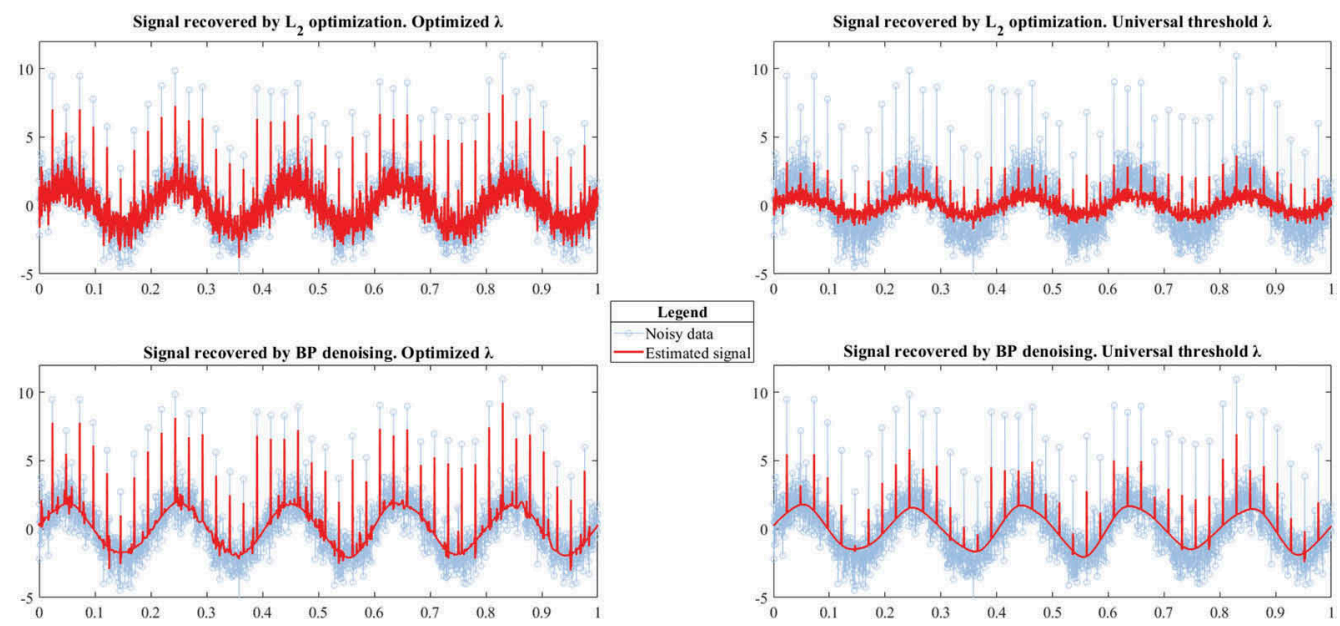

Figure 2. Simulated signal reconstruction results. Left: $\mathrm{BP}$ [ $\mathrm{L}_{1}$ regularization], right: $\mathrm{L}_{2}$ regularization.
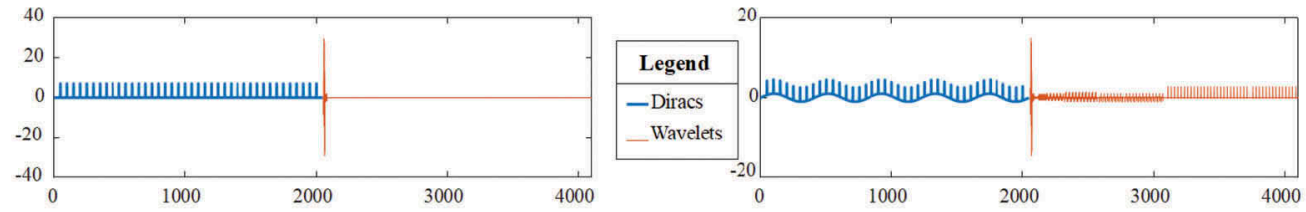

Figure 3. Components from Dirac and Wavelet basis used in signal reconstruction. Left: BP $\left[\mathrm{L}_{1}\right.$ regularization], right: $\mathrm{L}_{2}$ regularization.

The graphical results presented above highlight the evident advantage of $\mathrm{BP}\left[\mathrm{L}_{1}\right.$ regularization] over $\mathrm{L}_{2}$ regularization (Hastie et al., 2009): the BP-recovered signal is composed of fewer non-zero components than the $\mathrm{L}_{2}$-recovered signal, which eases interpretation, and the $\mathrm{BP}$ reconstruction also boasts smaller mean square error (MSE) than the reconstruction by $\mathrm{L}_{2}$ regularization. Besides, $\mathrm{BP}$ outperforms $\mathrm{L}_{2}$-regularization in terms of components from the two bases used to reconstruct the true signal - the MSE plummets to a constant, close-to-zero value after about 60 components are used, whereas the $\mathrm{L}_{2}$ regularization requires roughly as many components as the signal length (about 2000 components) to achieve the same MSE value (Figure 4).

\section{BASIS PURSUIT APPLIED TO REAL TSD DATA}

In Scavone et al. (in press), it is demonstrated that the TSD is capable of detecting the pulse response from structurally deficient joints in jointed pavements and that BP can extract such responses from a noisy TSD signal. In this chapter, we further elaborate on this subject, providing an example of how this technique could be applied in practice when only one TSD measurement is available.

In July 2019, a TSD surveyed Interstate 66 in the Washington, DC metropolitan area, as part of the network-wide TSD surveying program from the ongoing FHWA TPF-5-385 project. The selected pavements are the eastbound and westbound segments of I-66 between mile markers $[\mathrm{MM}] 52$ and 58, whose pavements are non-overlaid jointed concrete. The spacing between transverse joints is around 15 feet. The TSD operator [ARRB Inc.] provided georeferenced deflection slope and deflection bowl measurements for all segments at a 1-meter 


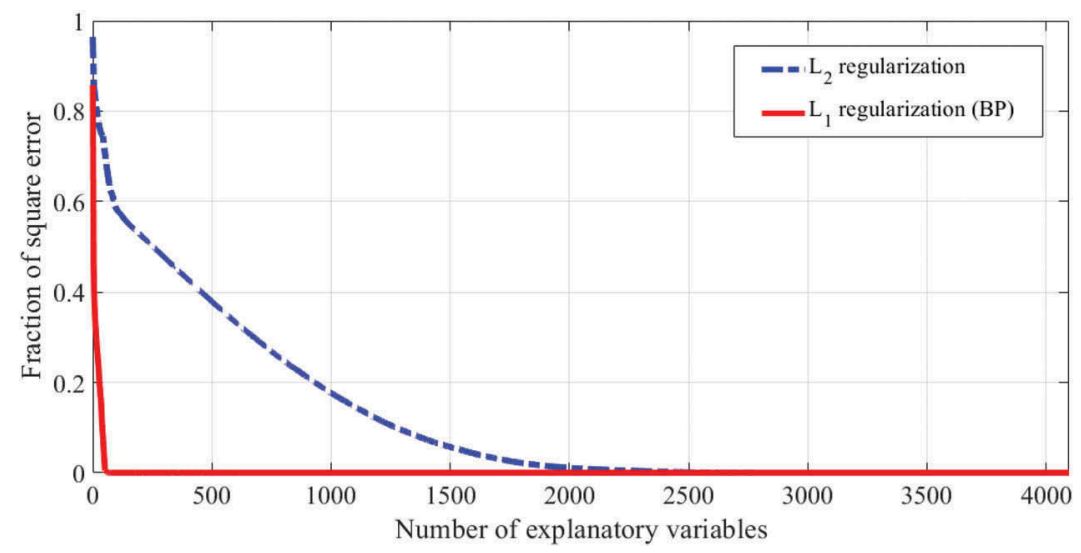

Figure 4. Decay of reconstruction error versus the number of non-zero components used in the reconstruction. Comparison for $\mathrm{L}_{1}$ and $\mathrm{L}_{2}$ regularization.

resolution, only one pass of the TSD over each segment was recorded. The TSD data was segmented as two subsections for each direction of travel (namely sections between MM 52-55 and 55-58), so, for the sake of analysis, four signals were processed independently. Each deflection measurement is tied to a picture of the pavement surface taken at that exact location using a downward-facing camera mounted on the TSD.

Signal denoising by BP requires the standard deviation of the measurement noise to be known. Yet, since the TSD ran only once over each segment, the signal's noise standard deviation can not be computed directly (as would happen when repeated measurements are available, like in the case study presented in Scavone et al. (2021). Instead, it has been approximated using the robust estimator presented in Katicha et al. (2015):

$$
\hat{\sigma}_{\text {noise }}=1.4826 \times \text { median }(x-\text { median }(x))
$$

A Matlab implementation of BP that processes each segment separately and extracts the recovered pulse components and their geographical coordinates was written for this case study. Processing the four segments required roughly 3 minutes on an average-spec personal computer. Figure 5 below shows the denoised TSD signal and the recovered pulse responses for one of these segments, both using the optimized penalty and the Universal Threshold.

The recovered pulse components were exported to a GIS application (QGis 2.18) for easy visualization and interpretation. The map in Figure 6 presents the detected weak spots with an amplitude greater than 300 microns for the EB and WB segments between MM 52 and 54 for the reconstruction with optimized $\lambda$ Those weak spots also detected by the BP decomposition with the universal threshold are highlighted with a circle. The 300 microns threshold used in the map was selected purposefully as it is roughly equal to the product between the Universal Threshold [about 4.20] and the TSD signal noise's standard deviation [about 64 microns], any recovered pulse with an amplitude lower than this threshold has chances of being a false positive, and any pulse with an amplitude higher than 300 microns has a high probability of being a true pulse representative of a location with a structural deficiency, thus worth highlighting for investigatory purposes.

Figure 7 Presents pictures of the pavement surface at or near high-probability weak spots, the point number corresponds to the point tags in Figure 6. Cases a) and b) represent actual locations where a pulse response was detected: For case a) the pulse response could have been produced either by the transverse joint or by the circular marking (probably the cut for a coil), while for case b) it is most likely that the (weak) transverse joint shown in the picture 

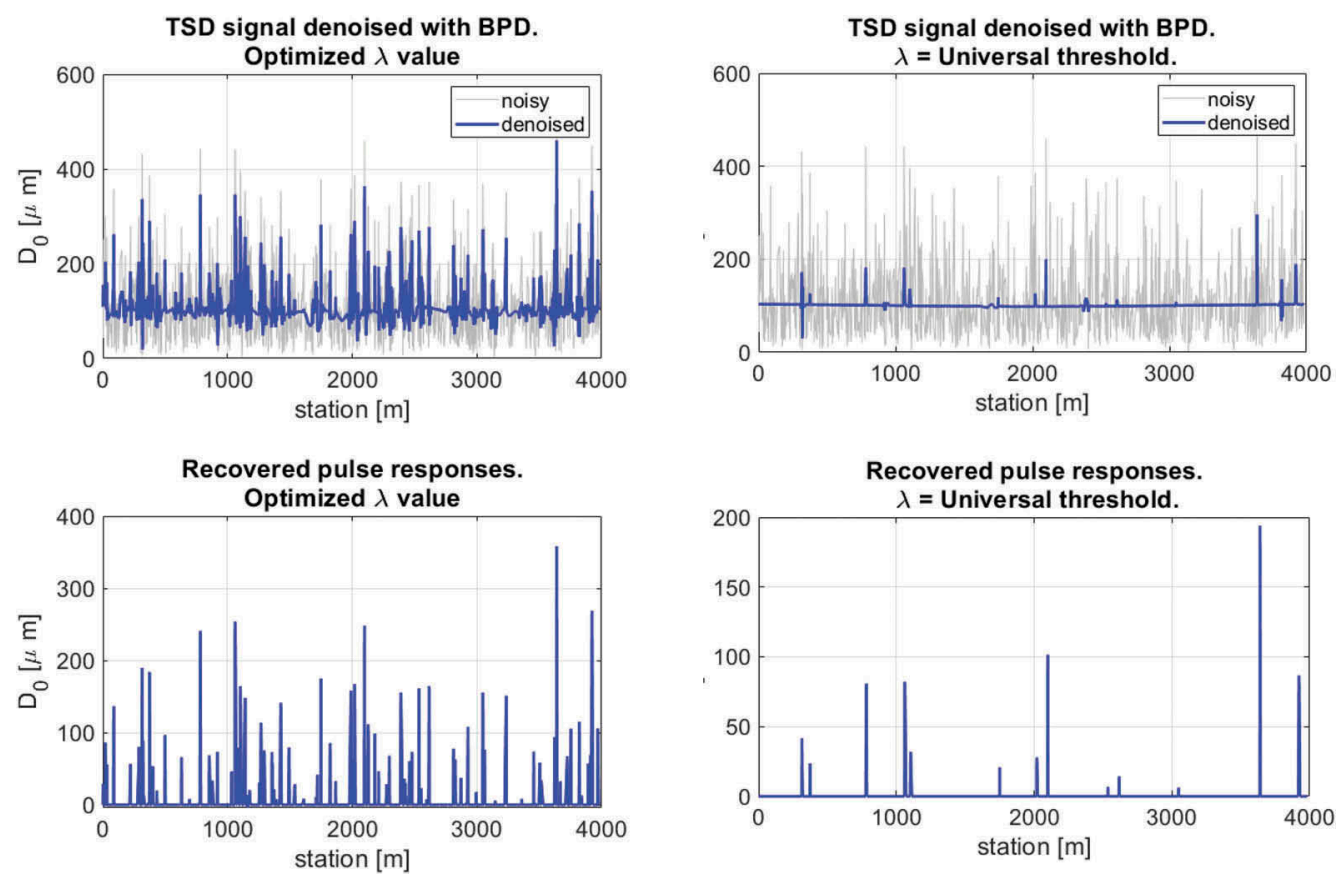

Figure 5. TSD signal recovered by BP I-66 eastbound between MM 52 and MM 55. Left plots: results for optimized penalty parameter $\lambda$, right plots: results for Universal Threshold.

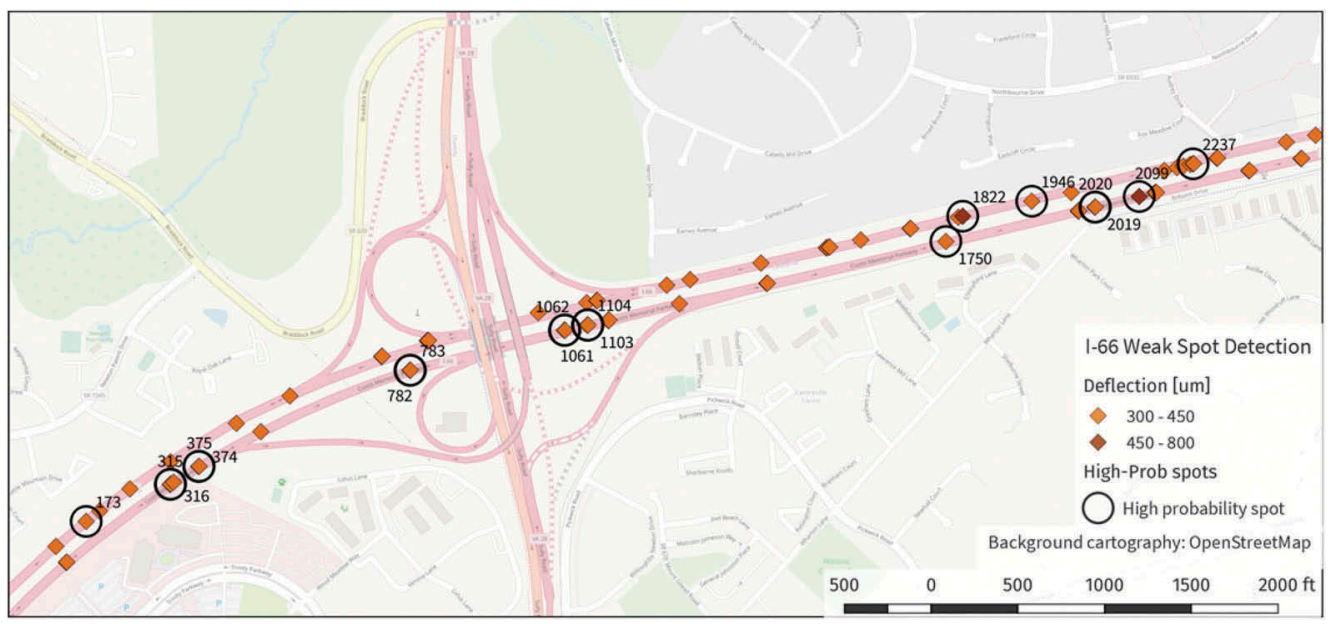

Figure 6. Interstate-66, MM 52-54. Weak spots pulse responses recovered by BPD.

be the culprit - although no surface distress developed in the concrete slabs. Meanwhile, picture c) represents the pavement 10 meters ahead of the weak joint shown in b), a joint in good structural condition for no pulse response was recorded by the TSD.

The remarkable result from this example is that the TSD could flag a potentially weak joint before any surface pathology related to lack of support or low LTE developed in the slab from the surface, the pavement at locations b) and c) looks in good condition, free of surface defects, yet one location is structurally deficient according to the TSD data. From 


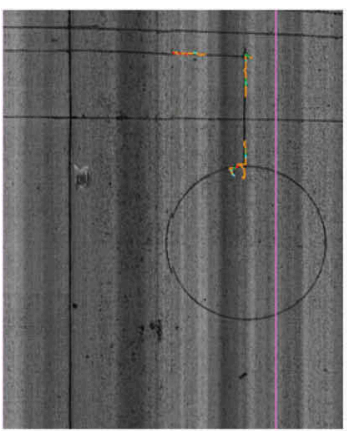

a) I66 WB, point 173 . Joint and cut for coil

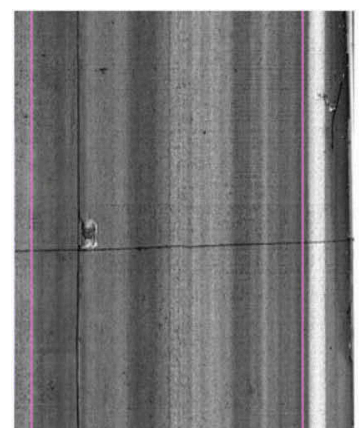

b) I66 EB, point 1061. Weak joint

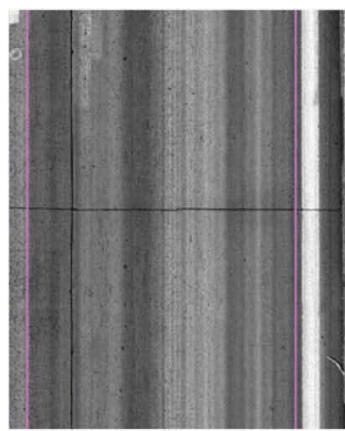

c) I66 EB, ahead of point 1061. Good joint

Figure 7. Pavement surface at selected weak spots. Imagery from the TSD.

a prevention standpoint, this is obviously advantageous as contributes to both rapidly assessing all the joints in the segment and pinpointing those with deficiencies before the lack of LTE takes its toll on the connecting slabs.

\section{CONCLUSIONS}

The TSD has been originally intended as a screening tool to pinpoint the location of structurally deficient points within a pavement network for investigatory purposes. This paper discusses a data analysis technique (Basis Pursuit, [BP]) to achieve this task in practice: BP analysis of the TSD measurements decomposes such measurements as a combination of elementary signals with an actual meaning or, in other words, that can be interpreted from an engineering perspective. The two examples presented in this paper demonstrate the effectiveness of the TSD to recognize structurally deficient joints within a concrete pavement segment even before the concrete slabs develop distress. The positive impact of such valuable information for preventive management purposes is evident, as it enables the manager to direct early corrective maintenance activities at those locations, saving the structure from developing further damage.

Nonetheless, there is still room for improvement in BP analysis of TSD signals: Reconstruction of signals by BP involves bias/variance trade-off, the penalty parameter $\lambda$ drives the reconstruction either towards less bias (at the cost of adding false-positive components to the reconstruction, fitting noise) or towards a reconstruction using high-probability components only (but with a damped recovered signal). Improvements to the reconstruction procedure were proposed by Candès et al. (2008) and Javanmard and Montanari (2018). If these lead to unbiased recovered components, further analyses could be made on the structural issues that are causing such pulse responses through back-calculation, as is proposed for instance by Deep et al. (2020). These ramifications are yet to be explored but open draw a promising range of applications for high-speed deflection devices.

\section{ACKNOWLEDGMENTS}

The authors would like to express their gratitude towards J. Daleiden and N. Bech from ARRB Inc., for providing the processed TSD data utilized in the case study presented herein, and to B. Diefenderfer from VDOT for his contribution to the I-66 case study. 


\section{REFERENCES}

Alavi, S.; LeCates, J. F.; Tavares, M. P. (2008): Falling Weight Deflectometer Usage. NCHRP Synthesis 381. Transportation Research Board.

American Association of State Highway and Transportation Officials (AASHTO) (2015): MechanisticEmpirical Pavement Design Guide. A Manual of Practice Publication Code MEPDG-2.

Andersen, S., Levenberg, E., \& Andersen, M. B. (2017). Inferring pavement layer properties from a moving measurement platform. In 10th International Conference on the Bearing Capacity of Roads, Railways and Airfields (pp. 675-682). Taylor \& Francis.

Arora, J. ., Tandon, V., \& Nazarian, S. (2006). Continuous deflection testing of highways at traffic speeds (No. FHWA/TX-06/0-4380-1).

Austroads (2016): Pavement Data Collection with a Traffic Speed Deflectometer (TSD) Device. Austroads Test Method AG:AM/T017. September 2016.

Candes, E. J., Wakin, M. B., \& Boyd, S. P. (2008). Enhancing sparsity by reweighted $\ell_{1}$ minimization. Journal of Fourier analysis and applications, 14(5-6), 877-905.

Chen, S. S., Donoho, D. L., \& Saunders, M. A. (2001). Atomic decomposition by basis pursuit. SIAM Review, 43(1), 129-159.

Deep, P., Andersen, M. B., Rasmussen, S., Marradi, A., Thom, N. H., \& Presti, D. L. (2020). Simulating Deflection of a Jointed Rigid Pavement Under Rolling Wheel Deflectometer (RAPTOR) Loading. In Proceedings of the 9th International Conference on Maintenance and Rehabilitation of PavementsMairepav9 (pp. 859-870). Springer, Cham.

Delatte, N. J. (2014). Concrete pavement design, construction, and performance. CRC Press.

Donoho, D. L., \& Johnstone, J. M. (1994). Ideal spatial adaptation by wavelet shrinkage. Biometrika, 81 (3), 425-455.

Flintsch, G. W.; Katicha, S. W.; Bryce, J.; Ferne, B.; Nell, S.; Diefenderfer, B. (2013): Assessment of Continuous Pavement Deflection Measuring Technologies. SHRP-2 Report S2-R06F-RW-1. Transportation Research Board, Washington DC.

Friedman, J., Hastie, T., \& Tibshirani, R. (2010). Regularization paths for generalized linear models via coordinate descent. Journal of statistical software, 33(1), 1.

Haas, R.; Hudson, W. R.; Falls, L. C. (2015): Pavement Asset Management. First Edition. Scrivener Publishing.

Hastie, T., Tibshirani, R., \& Friedman, J. (2009). The elements of statistical learning: data mining, inference, and prediction. Springer Science \& Business Media.

Huang, Y. H. (2004). Pavement Analysis and Design. Second Edition. Pearson.

Javanmard, A., \& Montanari, A. (2018). Debiasing the Lasso: Optimal sample size for gaussian designs. The Annals of Statistics, 46(6A), 2593-2622.

Katicha, S. W.; Flintsch, G. W.; Ferne, B. (2013): Optimal Averaging and Localized Weak Spot Identification of Traffic Speed Deflectometer Measurements. Transportation Research Record 2367, 43-52.

Katicha, S. W.; Flintsch, G. W.; Bryce, J; Ferne, B. (2014): Wavelet Denoising of TSD Deflection Slope Measurements for Improved Pavement Structural Evaluation. Computer-Aided Civil and Infrastructure Engineering 00, 1-17.

Katicha, S. W.; Flintsch, G. W.; Ferne, B.; Bryce, J. (2014): Limits of Agreement Method for Comparing TSD and FWD Measurements. International Journal of Pavement Engineering, 15(6), 532-541.

Katicha, S. W., Bryce, J., Flintsch, G., \& Ferne, B. (2015). Estimating "True" Variability of Traffic Speed Deflectometer Deflection Slope Measurements. Journal of Transportation Engineering, 141(1), 04014071

Katicha, S. W.; Loulizi, A.; El Khouri, J.; Flintsch, G. W. (2016): Adaptive False Discovery Rate for Wavelet Denoising of Pavement Continuous Deflection Measurements. Journal of Computing in Civil Engineering, 31(2), 04016049-1-10

Pierce, L.; Uhlmeyer, J.; Weston, J.; Lovejoy, J.; Mahoney, J. P. (2003): Ten-Year Performance of Dowel-Bar Retrofit. Application, Performance, and Lessons Learned. Transportation Research Record 1853, 83-91

Rada, G.; Nazarian, S.; Visintine, B. A.; Siddhartan, R.; Thyagarajan, S. (2016): Pavement Structural Evaluation at the Network Level: Final Report. (FHWA-HRT-15-074). United States Department of Transportation, Federal Highway Administration. September 2016

Scavone, M. A.; Katicha, S. W.; Flinstch, G. W. (2021): Identifying Weak Joints in Jointed Concrete and Composite Pavements from Traffic Speed Deflectometer Measurements by Basis Pursuit. Journal of Computing in Civil Engineering. DOI: 10.1061/(ASCE)CP.1943-5487.0000951 
Steele, D. A.; Lee, H.; Beckemeyer, C. A. (2020): Development of the Rolling Wheel Deflectometer $(R W D)$. (FHWA-DTFH-61-14-H00019). United States Department of Transportation, Federal Highway Administration. March 2020

Stein, C. M. (1981). Estimation of the mean of a multivariate normal distribution. The Annals of Statistics, 1135-1151.

Tibshirani, R. (1996). Regression shrinkage and selection via the Lasso. Journal of the Royal Statistical Society: Series B (Methodological), 58(1), 267-288.

Tibshirani, R. J., \& Taylor, J. (2012). Degrees of freedom in Lasso problems. The Annals of Statistics, 40 (2), 1198-1232. 